\title{
Feasibility of lentiviral-mediated sodium iodide symporter gene delivery for the efficient monitoring of bone marrow-derived mesenchymal stem cell transplantation and survival
}

\author{
SHUO SHI, MIN ZHANG, RUI GUO, YING MIAO, MIAO ZHANG, JIAJIA HU, YUN XI and BIAO LI \\ Department of Nuclear Medicine, Ruijin Hospital, Shanghai Jiaotong University \\ School of Medicine, Shanghai 200025, P.R. China
}

Received June 22, 2014; Accepted September 16, 2014

DOI: $10.3892 / \mathrm{ijmm} .2014 .1970$

\begin{abstract}
The aim of the present study was to explore the feasibility of lentiviral-mediated sodium iodide symporter (NIS) gene delivery for monitoring bone marrow-derived mesenchymal stem cell (BMSC) transplantation into the infarcted myocardium. For this purpose, we constructed a lentiviral vector (Lv-EF1 $\alpha$-NIS-IRES-EGFP) expressing NIS and enhanced green fluorescent protein (EGFP), and introduced it into BMSCs at different multiplicities of infection (MOI). The expression of EGFP was observed under a fluorescence microscope. Iodine uptake and the inhibition of iodine uptake by sodium perchlorate $\left(\mathrm{NaClO}_{4}\right)$ in the Lv-EF1 $\alpha$-NIS-IRES-EGFP-treated BMSCs were dynamically monitored in vitro. The Lv-EF1 $\alpha$-NIS-IRES-EGFP-treated BMSCs were transplanted into the infarcted myocardium of Sprague-Dawley rats, and ${ }^{99 \mathrm{~m}} \mathrm{Tc}^{99 \mathrm{~g}}$ (Tc, technetium; $99 \mathrm{~m}$ indicates that technetium is at its excited stage; $99 \mathrm{~g}$ indicates the atomic weight of technetium) micro-single-photon emission computed tomography (SPECT)/computed tomography (CT) imaging was performed in vivo 1 week following transplantation. The isolated BMSCs successfully differentiated into adipocytes and osteoblasts. The BMSCs were positive for the cell surface markers, CD105, CD29 and CD90, and negative for CD14, CD34 and CD45. Lv-EF1 $\alpha$-NIS-IRES-EGFP was efficiently transfected into the BMSCs. RT-qPCR and western blot analysis confirmed that the BMSCs expressed high protein and mRNA levels of NIS by day 7 following infection, and NIS expression remained at a consistent level from day 14 to 21 . In the Lv-EF1 $\alpha$-NIS-IRES-EGFP-treated BMSCs, the accumulation of iodine-125 ( $\left.{ }^{125} \mathrm{I}\right)$ was observed in vitro and was successfully monitored by ${ }^{99 \mathrm{~m}} \mathrm{Tc}^{99 \mathrm{~g}}$ micro-SPECT/CT imaging at 1 week following transplantation. These results suggest that lentiviral vectors are powerful vehicles for studying gene delivery in
\end{abstract}

Correspondence to: Professor Biao Li, Department of Nuclear Medicine, Ruijin Hospital, Shanghai Jiaotong University School of Medicine, 197 Ruijin Er Rd, Shanghai 200025, P.R. China

E-mail:1b10363@rjh.com.cn

Key words: lentiviral vector, bone marrow-derived mesenchymal stem cells, sodium iodide symporter, iodide, molecular imaging
BMSCs. It is feasible to use lentiviral vectors to deliver an NIS gene for the non-invasive monitoring of BMSC transplantation and survival in the infarcted myocardium in vivo.

\section{Introduction}

Bone marrow-derived mesenchymal stem cells (BMSCs) can be easily isolated and expanded from bone marrow aspirates. BMSCs are promising sources for regenerative medicine as they are harvested directly from patients (1). BMSCs are immunomodulatory, suppressing mixed lymphocyte reactions and attenuating alloresponses (2). Moreover, they are multipotent progenitor cells that have the capacity to differentiate into various types of cells, such as bone, cartilage, muscle, endothelial, vascular smooth muscle and other connective tissues (3). Due to their broad capacity for differentiation, BMSCs have been tested in multiple diseases. including diseases of the nervous system (4), skeletal (5) and renal system (6), and have been extensively used in myocardial disease $(7,8)$. A previous study reported that BMSCs can produce a variety of cytokines, including vascular endothelial growth factor, basic fibroblast growth factor, interleukin-1, platelet-derived growth factor and transforming growth factor, which contribute to the functional improvement of infarcted hearts by inhibiting the apoptosis of cardiomyocytes and inducing therapeutic angiogenesis (9). The potential of therapy using BMSCs to differentiate into viable cardiomyocytes and regenerate vascularization is, therefore, an attractive prospect, with the aim of reversing ventricular remodeling, preventing heart failure and alleviating the need for heart transplantation. Pre-clinical studies found that the implantation of BMSCs to the infarct zone in the heart improved the wall thickness of the left ventricle (LV), and promoted neo-vascularization in a rat model (7). In addition, implantation significantly increased LV function, cardiac blood flow and vascular density in a pig model (10). However, only a small fraction of transplanted cells engraft and survive in the injured heart, which limits the efficacy of cell transplantation (11). A clinical study demonstrated that BMSC intracoronary transplantation in patients with anterior acute myocardial infarction did not result in an increase in ejection fraction, although slight improvements in myocardial perfusion were noted in the BMSC group (12). Advanced imaging technologies are essential for 
the pre-clinical evaluation of novel cell-based therapeutics, as they permit longitudinal tracking and monitoring of cellular grafts and donor cell survival, which provides a more detailed understanding of the mechanisms involved in stem cell transplantation in ischemic heart disease.

To date, the majority of studies on stem cell viability have relied on ex vivo analysis, such as histological staining for green fluorescent protein or $\beta$-galactosidase. Another approach is to label cells with iron particles and track cell viability by magnetic resonance imaging (MRI) (13). MRI, however, is unable to distinguish viable from non-viable cells, as iron particles may be retained by living, dead, or scavenger cells (14). Another promising approach is based on the transfer of a sodium iodide symporter (NIS) reporter gene construct into stem cells using a viral vector (15), which permits the detection of viable transplanted cells by positron emission tomography (PET) or single photon emission computed tomography (SPECT) following iodine-125 $\left({ }^{125} \mathrm{I}\right)$ or ${ }^{99} \mathrm{Tc}^{99 \mathrm{~g}}$ ( $\mathrm{Tc}$, technetium; $99 \mathrm{~m}$ indicates that technetium is at its excited stage; $99 \mathrm{~g}$ indicates the atomic weight of technetium) radiotracer administration (16). A number of studies have successfully introduced the ectopic expression of NIS for non-invasive imaging analyses $(17,18)$.

In the current study, we employed a lentiviral vector to induce the expression of the NIS reporter gene and enhanced green fluorescence protein (EGFP) in BMSCs. The potential of NIS as an imaging reporter gene for the uptake and accumulation of ${ }^{125} \mathrm{I}$ and ${ }^{99 \mathrm{~m}} \mathrm{Tc}^{99 g}$ in vitro and in vivo was investigated using a rat model of ischemia.

\section{Materials and methods}

Animals. Sprague-Dawley rats were obtained from Slaccas Experimental Animal Corp. (Shanghai, China). Animal studies were approved by the local Ethics Committee (Shanghai Jiaotong University School of Medicine) and performed according to ethical principles of animal experimentation. All animals were anesthetized with pentobarbital $(100 \mathrm{mg} / \mathrm{kg}, 1$ dose intraperitoneally) prior to sacrifice. The efficacy of the anesthesia was monitored by pinching the hind paw. When sufficiently sedated, the rats were euthanized by cervical dislocation.

BMSC isolation and culture conditions. Four-week-old male Sprague-Dawley rats (weighing $80 \pm 5 \mathrm{~g}$ ) were used for BMSC isolation. The BMSCs were harvested, propagated and characterized as previously described (19). Briefly, both ends of the femur were cut off at the epiphysis, and the BMSCs were flushed out from the femurs and tibias with Dulbecco's modified Eagle's medium (DMEM; Gibco-BRL, NY, USA) containing $23 \mathrm{mM} \mathrm{NaHCO}$ (Gibco Biocult, Paisley, UK) and supplemented with $10 \%$ heat-inactivated fetal bovine serum (FBS; Gibco Biocult) and antibiotics $(50 \mu \mathrm{g} / \mathrm{ml}$ streptomycin sulfate and $100 \mathrm{U} / \mathrm{ml}$ penicillin). The cells were cultured in DMEM at $37^{\circ} \mathrm{C}$ in a humidified $5 \% \mathrm{CO}_{2}$ incubator.

Virus production and cell culture. For the generation of transgenic BMSC lines, the lentiviral vector, Lv-EF1 $\alpha$-NISIRES-EGFP, was constructed. Lv-EF1 $\alpha$-OCT4-IRES-EGFP was kindly provided by the Institute of Molecular Biology, Chinese Academy of Sciences, Shanghai, China; pcDNA3.1NIS was obtained from our own library, as previously described (20). The NIS gene was amplified from pcDNA3.1NIS by PCR using the following primers: forward, 5'-GCGC GGATCCCGGGTATCGATGGAGGCCGTG-3' and reverse, 5'-CGCGTCTAGATCAGAGGTTTGTAGGTAGTGAGC-3'. The product was digested with $\mathrm{XbaI}$ and $\mathrm{BamHI}$, and cloned into the $X b a \mathrm{I}$ and BamHI sites of Lv-EF1 $\alpha$-OCT4-IRES-EGFP generating a functional vector featuring NIS under the control of the human elongation factor- $1 \alpha(\mathrm{EF} 1 \alpha)$ promoter, while the octamer-binding transcription factor 4 (OCT4) transgene of Lv-EF1 $\alpha$-OCT4-IRES-EGFP was replaced with NIS.

The HEK293T cell line (Cell Bank of the Chinese Academy of Sciences, Shanghai, China) was cultured in RPMI-1640 medium (Gibco-BRL) supplemented with 10\% FBS and $1 \%$ penicillin/streptomycin.

Viral particles were generated by co-transfection of the HEK293T cells with Lv-EF1 $\alpha$-NIS-IRES-EGFP and the 3 packaging plasmids, pRsv-REV, pMDIg-pRRE and pMD2G (Biovector Science Laboratory, Beijing, China). The viral particles were harvested by collecting the cell culture medium at $48 \mathrm{~h}$ post-transfection. The supernatants were filtered through $0.45-\mu \mathrm{m}$ filters, centrifuged at $10,000 \mathrm{x} \mathrm{g}$ for $15 \mathrm{~min}$ and the resulting pellet was resuspended in $100 \mu \mathrm{l}$ culture medium.

Gene transduction and cell viability assay. The Lv-EF1 $\alpha-\mathrm{NIS}-$ IRES-EGFP virus at various multiplicities of infection (MOI) from 10 to 1,200 was used to infect the BMSCs and the infection efficiency was detected by fluorescence microscopy of the expression of green fluorescent protein.

Following gene transduction, cell viability and proliferation were determined using the cell counting kit-8 (CCK-8) assay (Beyotime Institute of Biotechnology, Shanghai, China). Transduced or non-transduced BMSCs were plated into 96-well plates $\left(2 \times 10^{3}\right.$ cells/well $)$, and incubated for $12,24,36,48$ or $72 \mathrm{~h}$. The blank group contained medium without cells. CCK-8 reagent $(10 \mu \mathrm{l})$ was added to the wells, and the cells were incubated for $1 \mathrm{~h}$. Absorbance was measured using a Multiskan MK3 Microplate Reader (Thermo Fisher Scientific, Hudson, NH, USA) at $450 \mathrm{~nm}$. The absorbance was calculated as $A_{\text {test }}-A_{\text {blank }}$, where $A_{\text {test }}$ represents the measured absorbance of each experimental group, and $\mathrm{A}_{\text {blank }}$ represents absorbance of each blank group. The mean \pm standard deviation (SD) of quadruplicate replicates from at least 3 independent experiments are presented.

Phenotypic expression and differentiation of BMSCs. To verify the phenotype of the isolated BMSCs, the cells were examined for the expression of various surface markers (CD105, CD29, CD90, CD14, CD34 and CD45) characteristic of BMSCs by flow cytometry (Beckman Coulter, Miami, FL, USA). Following 3 passages, the cells were used for in vitro and in vivo experiments. The BMSCs were plated on 6-well plates at a density of $10^{5}$ cells per well and cultured in DMEM at $37^{\circ} \mathrm{C}$ in a humidified $5 \% \mathrm{CO}_{2}$ incubator. Adipogenic differentiation was induced by treating $50 \%$ confluent cultures twice weekly for 2 weeks with $10 \mathrm{nM}$ dexamethasone and $5 \mu \mathrm{g} / \mathrm{ml}$ insulin, as previously described (21). Osteocyte differentiation was induced by treating $50 \%$ confluent cultures twice weekly for 4 weeks with $10 \mathrm{nM}$ dexamethasone, $50 \mu \mathrm{g} / \mathrm{ml}$ ascorbic acid and $10 \mathrm{mM} \beta$-glycerol phosphate, as previously described (22). The cells were fixed for $20 \mathrm{~min}$ in $10 \%$ buffered formalin. Lipid droplets in the adipocytes were stained with Oil Red O $(0.5 \%$ 
in isopropropanol stock diluted $3: 2$ in $\mathrm{H}_{2} \mathrm{O}$ ) for $10 \mathrm{~min}$, and bone matrix was stained for $20 \mathrm{~min}$ in $2 \%$ alizarin red.

Quantitative reverse transcription $P C R(R T-q P C R)$ and western blot analysis. To examine the expression levels of NIS in the Lv-EF1 $\alpha$-NIS-IRES- EGFP-transfected BMSCs, RT-qPCR was performed on days $1,4,7,14$ and 21 following viral infection, and western blot analysis was performed on day 7. Total RNA was extracted using TRIzol reagent (Invitrogen, Carlsbad, CA, USA). cDNA was synthesized using the Superscript RT kit (Invitrogen). RT-qPCR was performed using SYBR ${ }^{\circledR}$ Premix Ex Taq ${ }^{\mathrm{TM}}$ II (Takara Bio, Inc., Shiga, Japan) according to the manufacturer's instructions. The primers used for amplification were as follows: NIS forward, 5-GTACATTGTAGCCACGAT GCTGTA-3' and reverse, 5'-CCGTGTAGAAGGTGCAGAT AATTC-3'; GAPDH (internal control) forward, 5'-GTCAAG CTCATTTCCTGGTATGAC-3' and reverse, 5'-CTCTCTC TTCCTCTTGTGCTCTTG- $3^{\prime}$ at $95^{\circ} \mathrm{C}$ for $30 \mathrm{sec}$ followed by 40 cycles of $5 \mathrm{sec}$ at $95^{\circ} \mathrm{C}$ and $30 \mathrm{sec}$ at $60^{\circ} \mathrm{C}$ and one cycles of $95^{\circ} \mathrm{C}$ for $15 \mathrm{sec}, 60^{\circ} \mathrm{C}$ for $1 \mathrm{~min}, 95^{\circ} \mathrm{C}$ for $15 \mathrm{sec}$. According to the manufacture's instructions, the NIS expression levels were normalized to those of the GAPDH endogenous reference gene as follows: $\mathrm{F}$ value $=2^{-\Delta \Delta \mathrm{Ct}}$, as previously described (23).

Total protein was harvested from the cultured cells on day 7 following viral infection. The cells were incubated in lysis buffer (SDS lysis buffer), 1\% phenylmethanesulfonyl fluoride (PMSF) on ice, centrifuged at $10,000 \times \mathrm{g}$, and the protein concentration of the supernatants was measured using the BCA Protein Assay kit (all from Beyotime Institute of Biotechnology). Equal quantities of protein were subjected to western blot analysis using a polyclonal goat anti-NIS antibody (1:500; Santa Cruz Biotechnology, Inc., Santa Cruz, CA, USA), and an anti-goat IgG-HRP secondary antibody (1:5,000; MultiSciences Biotech Co., Ltd., Shanghai, China). Relative protein levels were normalized against GAPDH (1:1,000; Beyotime Institute of Biotechnology). All experiments were performed in triplicate.

Analysis of ${ }^{125}$ I uptake and efflux. ${ }^{125}$ I uptake and efflux were measured in triplicate as previously described (24). On day 1, transduced or control BMSCs were plated in 24-well plates ( $2 \times 10^{5}$ cells/well). On day 2, $500 \mu \mathrm{l}$ of Hank's Balanced Salt Solution (HBSS) containing $3.7 \mathrm{kBq}{ }^{125} \mathrm{I}$ and $10 \mu \mathrm{mol} / 1$ sodium iodide (NaI) were added. The cells in the control group were treated with $10 \mu \mathrm{mol} / 1 \mathrm{NaI}$, whereas the cells in the test group were treated with $50 \mu \mathrm{M}$ sodium perchlorate $\left(\mathrm{NaClO}_{4}\right)$. The cells were incubated at $37^{\circ} \mathrm{C}$ for 5-120 min, washed twice with ice-cold HBSS, and lysed using $0.5 \mathrm{~mol} / 1$ sodium hydroxide $(\mathrm{NaOH})$. The radioactivity [counts per minute, $(\mathrm{CPM})]$ of the cell lysates was measured using an automatic gamma counter (Shanghai Hesuo Rihuan Photoelectric Instrument Co., Ltd., Shanghai, China).

In order to measure the efflux, the cells were incubated with $3.7 \mathrm{kBq} \mathrm{Na}{ }^{125} \mathrm{I}$ and $10 \mu \mathrm{M} \mathrm{NaI}$ in $500 \mu \mathrm{l}$ of $\mathrm{HBSS}$ at $37^{\circ} \mathrm{C}$ for $60 \mathrm{~min}$, washed twice with HBSS, and incubated in $500 \mu \mathrm{l}$ of HBSS containing $10 \mu \mathrm{M} \mathrm{NaI}$ (without radioactive $\mathrm{Na}^{125} \mathrm{I}$ ). The buffer was replaced every $5 \mathrm{~min}$ for up to $40 \mathrm{~min}$ and the level of radioactivity of the solutions was determined. Following the removal of the last sample, the cells were lysed using $0.5 \mathrm{M}$ $\mathrm{NaOH}$. Total radioactivity at the initiation of the measurement of the efflux was calculated by adding the final cell radioactivity to the total medium radioactivity.
Animal model of myocardial infarction. The experimental animals used in this study were male Sprague-Dawley rats, weighing 200-220 g. The rats were intraperitoneally anesthetized with pentobarbital $(35 \mathrm{mg} / 100 \mathrm{~g})$. A midline anterior cervical skin incision was made, and the trachea was exposed by sharp dissection. The trachea was intubated with an angiocatheter and ventilated to a rodent ventilator with room air. A $1.5 \mathrm{~cm}$ vertical left parasternal skin incision was made, the chest cavity was entered through the fourth interspace, and the pericardium was vertically opened. The left anterior descending (LAD) coronary artery was ligated with a 6-0 polypropylene suture. Ventricle blanching indicated the successful occlusion of the vessel.

Implantation of BMSCs. Adult male Sprague-Dawley rats were randomly divided into 2 groups. Immediately following the ligation of the LAD, the experimental group received $5 \times 10^{6} \mathrm{BMSCs}$ transfected with Lv-EF1 $\alpha$-NIS-IRES-EGFP; the control group received $5 \times 10^{6}$ BMSCs.

Micro-SPECT/computed tomography (CT) imaging. One week following BMSC transplantation, the rats were intravenously injected with $74 \mathrm{MBq}$ of ${ }^{99 \mathrm{~m}} \mathrm{Tc}^{99 \mathrm{~g}}$. Anesthesia was induced and maintained by isoflurane inhalation, and the rats were placed in a spread-prone position and scanned using a small-animal micro-SPECT scanner (NanoSPECT/CT ${ }^{\circledR}$ PLUS; Bioscan, Washington, DC, USA) $60 \mathrm{~min}$ after the injection of ${ }^{99 \mathrm{~m}} \mathrm{Tc}^{99 \mathrm{~g}}$. CT images were acquired (CTDI $=6.1 \mathrm{cGy}$ ) before whole-body NanoSPECT images (10 s/frame for systematic scans) were obtained, without moving the rats. The images were processed and reconstructed using Nuclear v1.02 software and HiSPECT 1.4.2 software (both from Bioscan) for image acquisition.

Histological analysis. After imaging, some animals from the Lv-EF1 $\alpha$-NIS-IRES-EGFP group were sacrificed by cervical dislocation. The hearts were harvested and immersed in $4 \%$ paraformaldehyde for $24 \mathrm{~h}$. The fixed hearts were sliced into 2 sections according to the injection sites. Heart sections containing the injection sites were then embedded in paraffin and cut into 2-3 $\mu \mathrm{m}$ sections. Hematoxylin and eosin (H\&E) staining was performed and unstained sections on positively charged slides were used for immunohistochemical staining using primary polyclonal rabbit anti-NIS antibody (1:50; Proteintech, Chicago, IL, USA).

Statistical analysis. Data were analyzed using GaphPad Prism software (version 5.0; GraphPad Software, Inc., San Diego, CA, $\mathrm{USA}$ ); the mean $\pm \mathrm{SD}$ values are presented. Statistical analyses were performed using two-tailed Student's t-tests. For all analyses, a value of $\mathrm{p}<0.05$ was considered to indicate a statistically significant difference.

\section{Results}

Immunophenotyping of BMSCs. The BMSCs were analyzed by flow cytometry and were found to be positive for the cell surface antigens, CD105, CD29 and CD90, and negative for CD14, CD34 and CD45 (Fig. 1A). The differentiation assay confirmed that the isolated BMSCs differentiated into adipocytes and osteoblasts. Lipid droplets in adipocytes were stained 

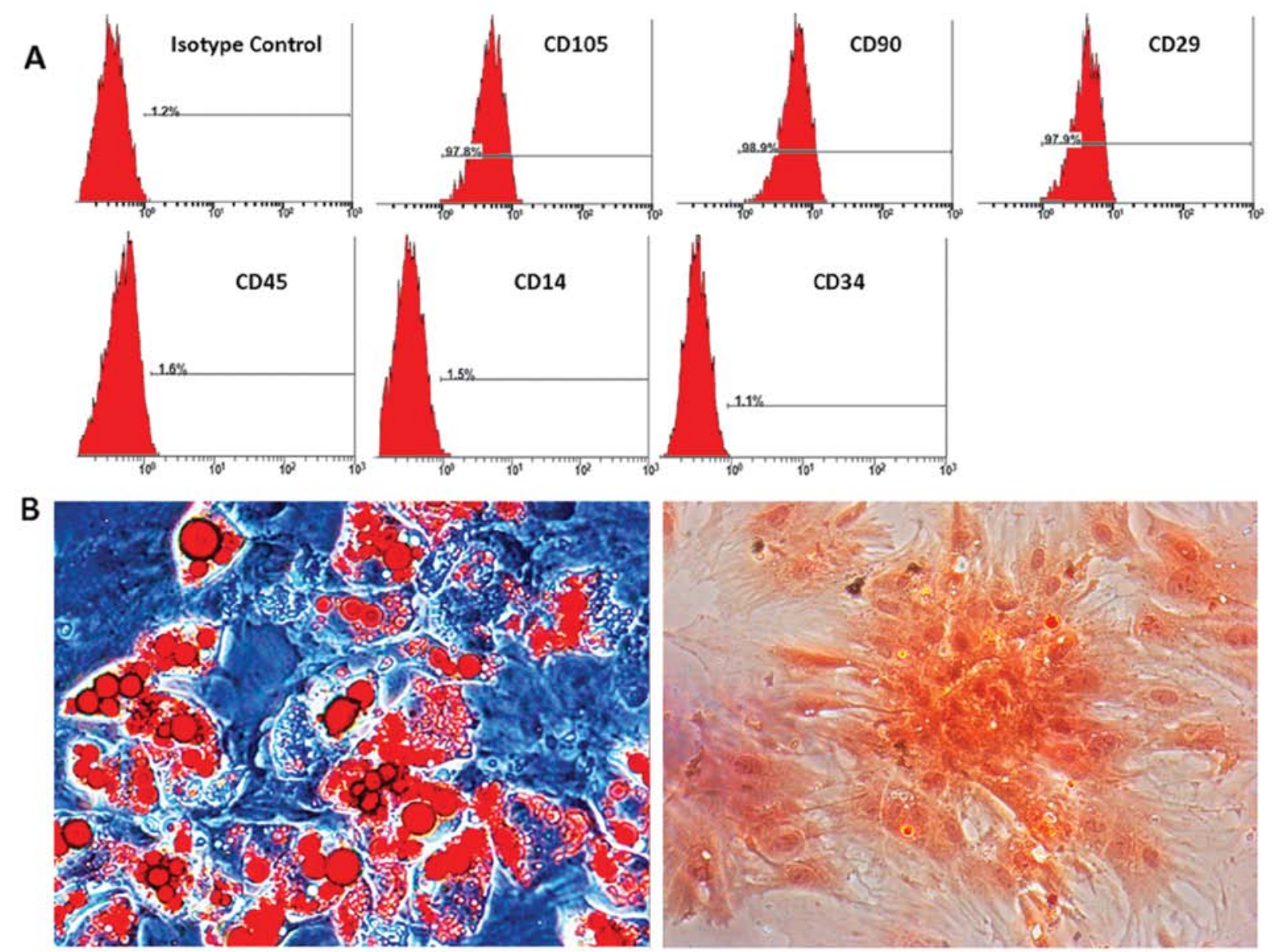

Figure 1. Isolation and identification of bone marrow-derived mesenchymal stem cells (BMSCs). (A) BMSCs were positive for CD105, CD29 and CD90 and negative for CD14, CD34 and CD45. (B) BMSCs differentiated into adipocytes (left panel) and osteoblasts (right panel).

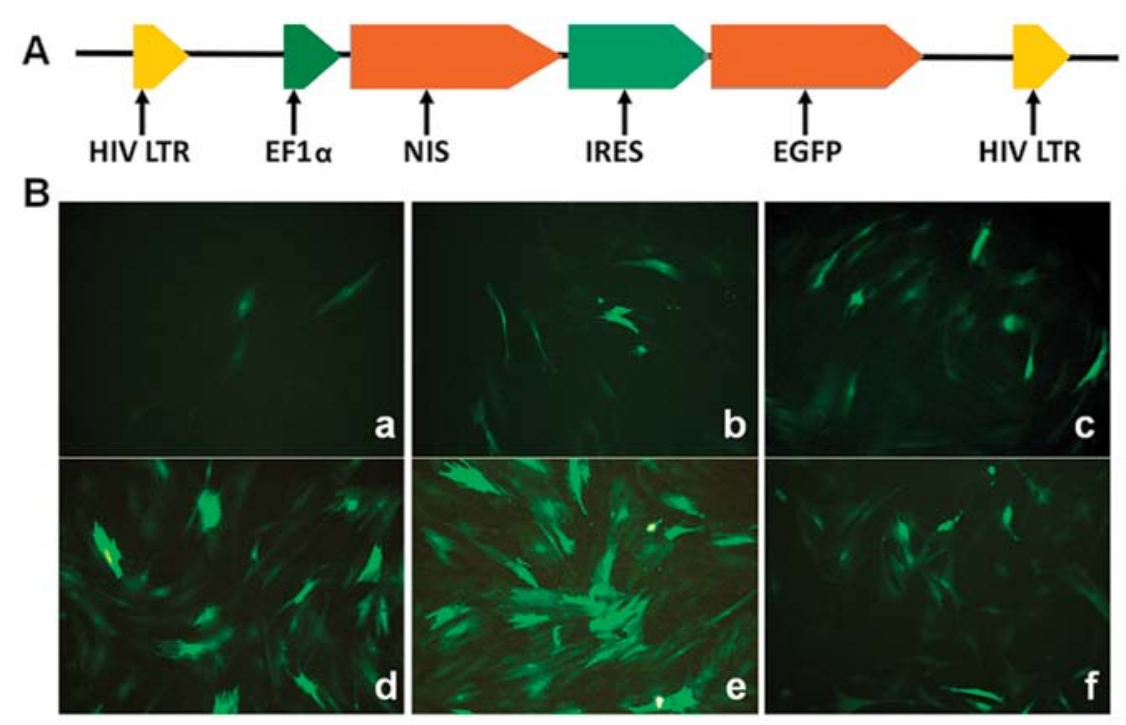

Figure 2. Construction of the Lv-EF1 $\alpha$-NIS-IRES-EGFP vector and determination of optimal multiplicities of infection (MOI). (A) Schematic representation of the reporter gene construct. The vector was designed for the transcription of sodium iodide symporter (NIS) and expression of enhanced green fluorescent protein (EGFP) using an internal ribosomal entry site (IRES). NIS was inserted between the elongation factor-1 $\alpha$ (EF1 $\alpha$ ) promoter and IRES using BamHI and $\mathrm{XbaI}$ restriction sites. (B) EGFP expression at different MOIs of Lv-EF1 $\alpha$-NIS-IRES-EGFP. (a) MOI, 10; (b) MOI, 50; (c) MOI, 100; (d) MOI, 400; (e) MOI, 600; (f) MOI, 1,200. At an MOI of 600,>90\% of the cells expressed EGFP.

red with Oil Red $\mathrm{O}$, and bone matrix was stained in orange red with alizarin red (Fig. 1B).
Infection with the lentiviral vector and determination of the optimal MOI. As illustrated in Fig. 2, the BMSCs were 

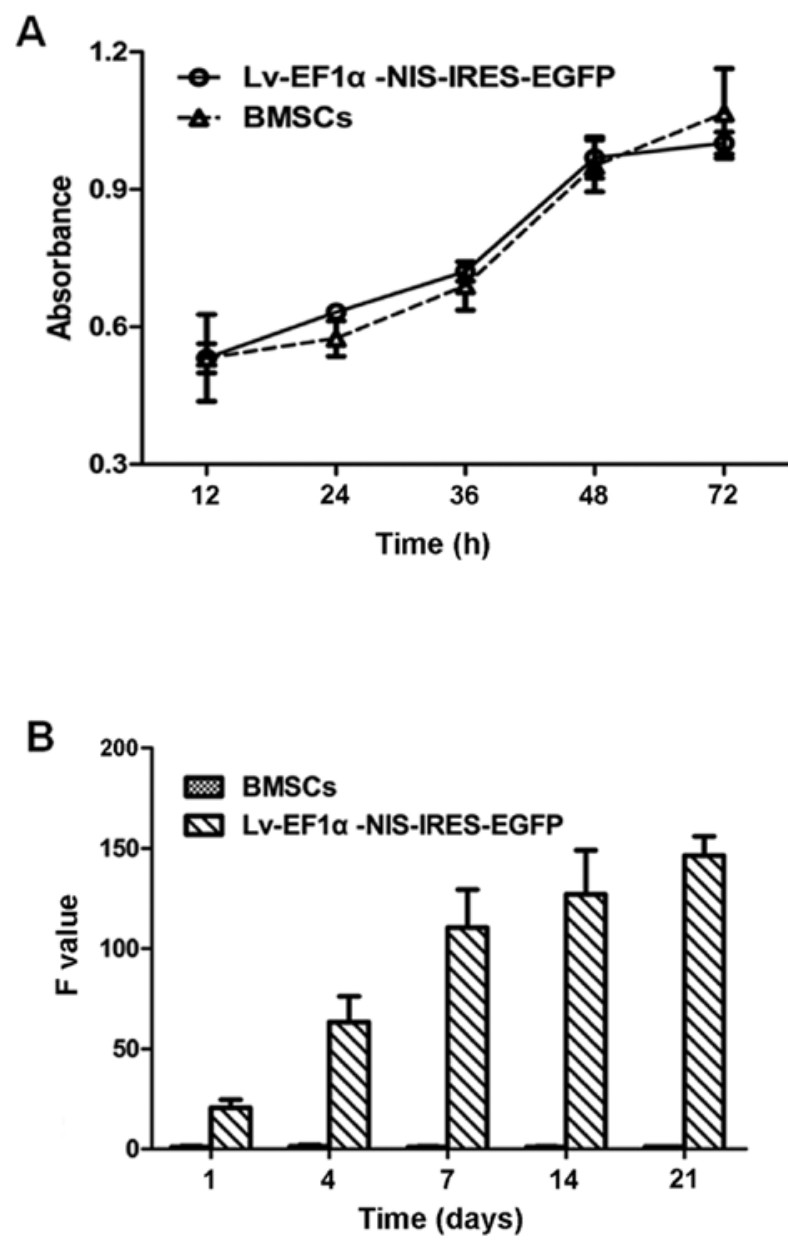

C

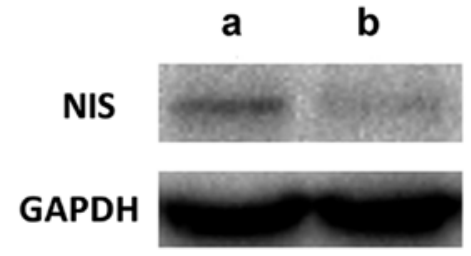

Figure 3. Effects of Lv-EF1 $\alpha$-NIS-IRES-EGFP on the proliferation of bone marrow-derived mesenchymal stem cells (BMSCs) and the detection of sodium iodide symporter (NIS) following gene transduction. (A) The proliferation of BMSCs infected with the lentivirus and those not infected at 12 , $24,36,48$ and $72 \mathrm{~h}$, as detected by the cell counting kit-8 (CCK-8) assay. The results are the means \pm standard deviation (SD) of 3 independent experiments. (B) mRNA expression of NIS in the Lv-EF1 $\alpha$-NIS-IRES-EGFP-infected and control BMSCs on days 1, 4, 7, 14 and 21. (C) NIS protein expression in the Lv-EF1 $\alpha$-NIS-IRES-EGFP-infected and control BMSCs groups on day 7. (a) Lv-EF1 $\alpha$-NIS-IRES-EGFP-infected BMSCs; (b) control (uninfected) BMSCs.

distributed uniformly at $48 \mathrm{~h}$ following infection with Lv-EF1 $\alpha$-NIS-IRES-EGFP at MOIs of 10, 50, 100, 400, 600 and 1,200 . The majority of the cells (>90\%) expressed EGFP at an MOI of 600 .

CCK-8 assay, RT-qPCR and western blot analysis. There was no significant difference in cell viability and proliferation between the BMSCs infected with Lv-EF1 $\alpha$-NIS-IRES-EGFP and the BMSC control group (Fig. 3A). To examine the expression levels of NIS in the BMSCs infected with Lv-EF1 $\alpha$-NIS-IRES-EGFP,
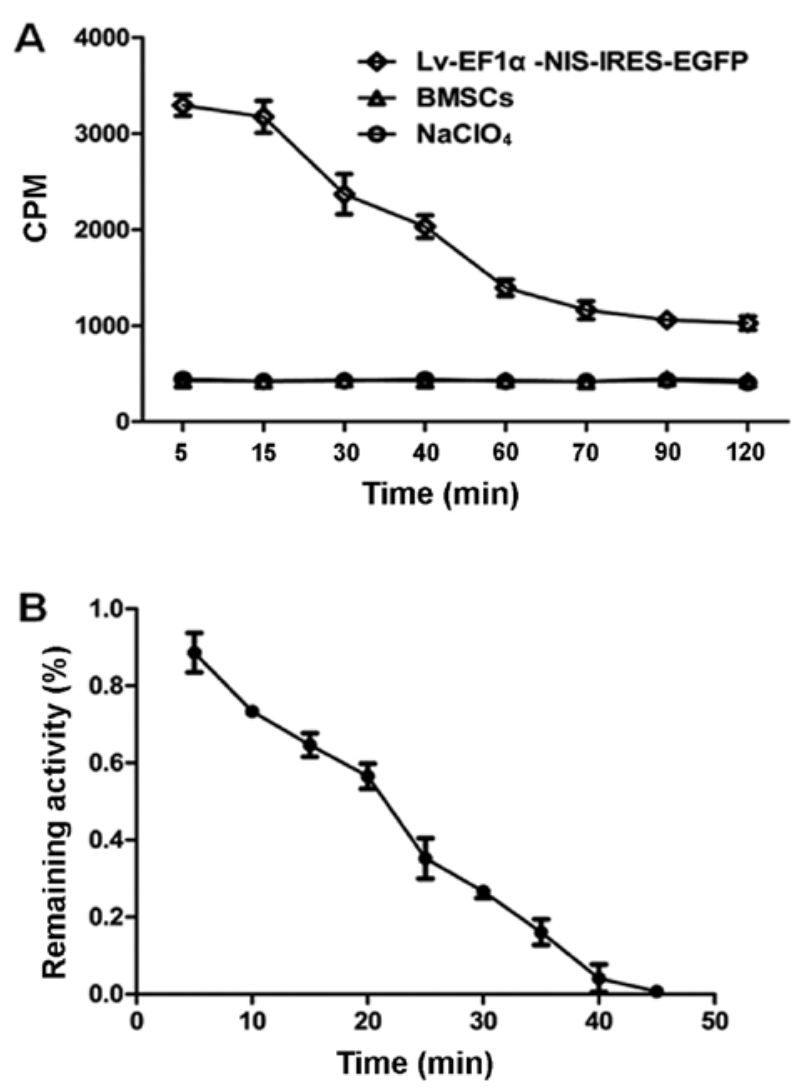

Figure 4. iodine-125 ( $\left.{ }^{125} \mathrm{I}\right)$ uptake and efflux. (A) The accumulation of ${ }^{125} \mathrm{I}$ in infected cells peaked at $5 \mathrm{~min}$. There was no accumulation of ${ }^{125} \mathrm{I}$ in the bone marrow-derived mesenchymal stem cell (BMSC) control group. Sodium perchlorate $\left(\mathrm{NaClO}_{4}\right)$ completely blocked ${ }^{125} \mathrm{I}$ uptake. (B) ${ }^{125} \mathrm{I}$ was rapidly effluxed from the Lv-EF1 $\alpha$-NIS-IRES-EGFP-infected cells, with a half life $\left(\mathrm{t}_{1 / 2}\right)$ of approximately $25 \mathrm{~min}$. CPM, counts per minute.

RT-qPCR was performed on days 1, 4, 7, 14 and 21 following viral infection and western blot analysis was performed on day 7. NIS mRNA and protein expression was clearly detected in the Lv-EF1 $\alpha$-NIS-IRES-EGFP-treated BMSCs compared with the control group (Fig. 3B and C). The results revealed that the expression of NIS increased from day 4 to 7 , and remained at a consistently high level from day 7 to 21 .

${ }^{125}$ I uptake and efflux. ${ }^{125}$ I uptake by the Lv-EF1 $\alpha-\mathrm{NIS}$ IRES-EGFP-infected cells varied depending on the incubation time, and peaked at approximately 3,300 CPM at $5 \mathrm{~min}$. This was 8 -fold higher than the level of ${ }^{125} \mathrm{I}$ uptake by the control BMSCs at the same time point. After 5 min, ${ }^{125}$ I uptake decreased with time. ${ }^{125}$ I uptake by the Lv-EF1 $\alpha$-NIS-IRES-EGFP-infected cells was completely blocked with $\mathrm{NaClO}_{4}$. There was no functional ${ }^{125} \mathrm{I}$ uptake observed in the control BMSC group (Fig. 4A). ${ }^{125} \mathrm{I}$ was rapidly effluxed from the Lv-EF1 $\alpha$-NIS-IRES-EGFP-infected BMSCs, with a half life $\left(\mathrm{t}_{1 / 2}\right)$ of approximately $25 \mathrm{~min}$ (Fig. 4B).

Micro-SPECT/CT imaging and immunostaining. One week following cell transplantation, the rats were intravenously injected with $74 \mathrm{MBq}$ of ${ }^{99 \mathrm{~m}} \mathrm{Tc}^{99 \mathrm{~g}} .{ }^{99 \mathrm{~m}} \mathrm{Tc}{ }^{99 \mathrm{~g}}$ uptake was not detectable in the heart, although significant uptake was observed in the stomach of the rats in the BMSC control 

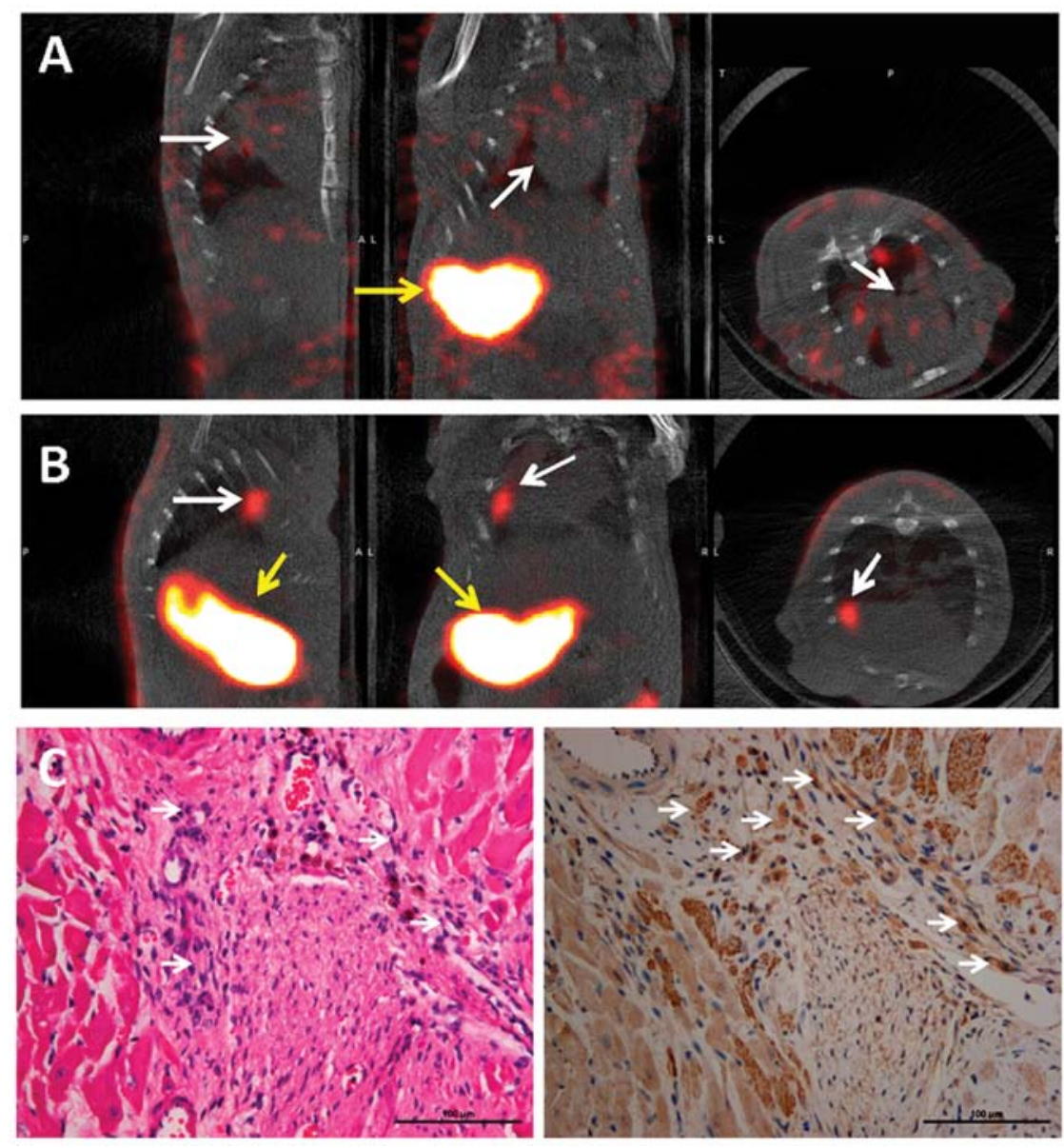

Figure 5.In vivo ${ }^{99 \mathrm{~m}} \mathrm{Tc}^{99 \mathrm{~g}}$ single photon emission computed tomography (SPECT)/computed tomography (CT) imaging of rats transplanted with bone marrow-derived mesenchymal stem cells (BMSCs) (control) orLv-EF1 $\alpha$-NIS-IRES-EGFP-infected BMSCs and immunostaining of transplantedLv-EF1 $\alpha$-NIS-IRES-EGFP-infected BMSCs. (A) Radioactive uptake in an animal from the control BMSC group 45 min after the injection of $74 \mathrm{MBq}^{99 \mathrm{~m}} \mathrm{Tc}^{999}$. Significant radioactive uptake was observed in the stomach (yellow arrow). (B) Radioactive uptake in an animal from the Lv-EF1 $\alpha$-NIS-IRES-EGFP group 45 min after the injection of $74 \mathrm{MBq}$ 99mTc99g. Significant radioactive uptake was observed in the transplanted zone of the heart (white arrow) and stomach (yellow arrow) of the rats transplanted with Lv-EF1 $\alpha$-NIS-IRES-EGFP-infected BMSCs (anesthesia was induced and maintained by isoflurane inhalation). (C, lef panel) Hematoxylin and eosin (H\&E) staining of BMSCs infected with the Lv-EF1 $\alpha$-NIS-IRES-EGFP virus (white arrow); (C, right panel) sodium iodide symporter (NIS) staining for transplanted BMSCs infected with the Lv-EF1 $\alpha$-NIS-IRES-EGFP virus (white arrow, NIS-positive BMSCs).

group (Fig. 5A). By contrast, significant uptake was observed in the transplanted zone of the hearts of rats transplanted with Lv-EF1 $\alpha$-NIS-IRES-EGFP-infected BMSCs and in the stomach 45 min following ${ }^{99}{ }^{9} \mathrm{Tc}^{99 g}$ injection (Fig. 5B). H\&E staining identified the transplanted cells in the infarct zone of the rat hearts (Fig. 5C, left panel). The BMSCs transfected with Lv-EF1 $\alpha$-NIS-IRES-EGFP were positive for NIS expression (Fig. 5C, right panel) .

\section{Discussion}

Despite rapid progress in the medical treatment of cardiometabolic disease, ischemic heart disease remains the leading cause of mortality in the developed world (25). Pre-clinical and clinical studies have demonstrated that BMSC transplantation into the infarcted myocardium can augment cardiac function and attenuate ventricular remodeling $(7,10,12,26)$. However, 99\% of BMSCs do not survive within 3-4 days following transplantation into the ischemic heart (27). In an attempt to prolong survival in vivo, genetically engineered BMSCs have been suggested as an effective strategy to improve the survival rate and therapeutic efficacy of BMSCs by inducing the expression of proteins, such as CXC chemokine receptor 4 (28), angiogenin (29), vascular endothelial growth factor (30), heme oxygenase-1 $(31,32)$, and hypoxia-inducible factor- $1 \alpha(8)$. The tracking and monitoring of transplanted cells relies on ex vivo analyses, such as histologic staining for green fluorescent protein or $\beta$-galactosidase or cellular labeling with Dil. These techniques require a large number of animals to be sacrificed and cannot be applied in clinical research. Advanced imaging technologies and non-invasive techniques are therefore be required. MRI has been used to track cell viability after labelling with iron particles (13). This technique, however, is unable to distinguish viable from non-viable cells, as iron particles may be retained by living, dead, or scavenger cells (14). NIS is a transmembrane carrier that selectively transports iodine (I), technetium $(\mathrm{Tc})$, rhenium $(\mathrm{Re})$ and their isotopes, ${ }^{123} \mathrm{I},{ }^{125} \mathrm{I},{ }^{131} \mathrm{I}$, ${ }^{99 \mathrm{~m}} \mathrm{Tc}^{99 \mathrm{~g}}$ and ${ }^{188} \mathrm{Re}(16,17,32)$, that can be detected by SPECT or PET, and offers several advantages for in vivo reporter gene imaging (33).

In this study, we evaluated the ability of the NIS reporter gene to monitor transplanted BMSCs in the ischemic myocar- 
dium of living rats. The technique involved inducing the expression of the NIS and EGFP genes, which was achieved with high efficiency at an MOI of 600 and without adverse effects following infection with a lentiviral vector driven by a single promoter, EF1 $\alpha$. The Lv-EF1 $\alpha$-NIS-IRES-EGFP lentiviral particles were successfully packaged and efficiently infected the BMSCs. The expression of NIS was confirmed by RT-qPCR and western blot analysis. To address concerns regarding the biosafety of the lentivirus, we assessed the effects of exogenous NIS expression on the viability and proliferation of BMSCs in vitro. However, no significant differences in cell viability or proliferation were measured in the control or treated BMSCs. The absorption and accumulation of ${ }^{125} \mathrm{I}$ was successfully observed in the BMSCs transfected with the lentivirus in vitro and was specifically inhibited by $\mathrm{NaClO}_{4}$. One week following BMSC transplantation, the BMSCs were successfully monitored by ${ }^{99} \mathrm{~m}^{\mathrm{T}}{ }^{99 \mathrm{~g}}$-SPECT.

A number of viruses have been used for gene transfer, and each has advantages and disadvantages. Herpes simplex virus has a broad infectivity, but low titers and short term episomal expression. Adenoviruses can be obtained with high titers and can infect non-dividing cells, but have life-threatening immunogenicity and short-term episomal expression. Adeno-associated viruses also have broad infectivity similar to the herpes simplex virus, infect non-dividing cells and are non-cytopathic; however, short-term expression with limited integration limits their broad use in gene transfer. Fortunately, lentiviruses can be obtained with high viral titers that can permanently infect non-dividing cells, although safety concerns exist due to their HIV derivation (34). To address this issue, the current packaging cell line requires transient transfection with three distinct plasmids, all containing gene sequences required for an active infectious virus. This system ensures that the possibility of three recombination events occurring in one cell to produce an actively infectious product is extremely unlikely (35). In our study, we used lentivirus for transfecting BMSCs to establish cell lines expressing the NIS and the EGFP genes which is useful for further study on BMSC viability and migration following transplantation into the infarcted myocardium.

NIS expression is limited to only a few tissues, such as the thyroid gland, salivary glands, stomach, lactating mammary glands, small intestine and rectum (36). A limitation of this imaging technology is the physiological expression of NIS in these tissues. If the signals from these organs are strong, they may cover up weak signals from adjacent organs of cell transplantation. In our study, significant radioactive uptake was observed in the transplanted Lv-EF1 $\alpha$-NIS-IRES-EGFP-treated BMSCs in the heart, stomach, urinary bladder, intestine and only a slight uptake in the thyroid in the Lv-EF1 $\alpha$-NIS-IRES-EGFP group at $45 \mathrm{~min}$ following ${ }^{99 \mathrm{~m}} \mathrm{Tc}^{99 \mathrm{~g}}$ injection. ${ }^{99 \mathrm{~m}} \mathrm{Tc}^{99 \mathrm{~g}}$ uptake of other tissues did not affect the signals from the transplanted BMSCs.

With rapid advances in genetically engineered stem cell-based therapy for myocardial infarction, non-invasive in vivo imaging may play a critical role in future studies. Our strategy of using lentivirus as a gene delivery vector in a radionuclide-based reporter gene imaging system may provide a valuable method for further studies on BMSC transplantation therapy for myocardial infarction.

\section{Acknowledgements}

This study was supported by grants from the National Natural Science Foundation of China (NSFC; no. 81271610), the Shanghai Outstanding Academic Leaders Project (11XD1403700), the Discipline Leaders Climbing Project of Ruijin Hospital and the Medical Engineering (Science) Cross micro-PET Special Foundation of Shanghai Jiaotong University (no. YG08PETZD01), national leading clinical discipline project.

\section{References}

1. Hess DC and Borlongan CV: Stem cells and neurological diseases. Cell Prolif 41 (Suppl 1): 94-114, 2008.

2. Jitschin R, Mougiakakos D, Von Bahr L, et al: Alterations in the cellular immune compartment of patients treated with third-party mesenchymal stromal cells following allogeneic hematopoietic stem cell transplantation. Stem Cells 31: 1715-1725, 2013.

3. Wang CH, Cherng WJ, Yang NI, et al: Late-outgrowth endothelial cells attenuate intimal hyperplasia contributed by mesenchymal stem cells after vascular injury. Arterioscler Thromb Vasc Biol 28: 54-60, 2008.

4. Shichinohe H, Kuroda S, Maruichi K, et al: Bone marrow stromal cells and bone marrow-derived mononuclear cells: which are suitable as cell source of transplantation for mice infarct brain? Neuropathology 30: 113-122, 2010.

5. Zou D, Zhang Z, Ye D, et al: Repair of critical-sized rat calvarial defects using genetically engineered bone marrow-derived mesenchymal stem cells overexpressing hypoxia-inducible factor-1 $\alpha$. Stem Cells 29: 1380-1390, 2011.

6. Liu N, Patzak A and Zhang J: CXCR4-overexpressing bone marrow-derived mesenchymal stem cells improve repair of acute kidney injury. Am J Physiol Renal Physiol 305: F1064-F1073, 2013.

7. Chi NH, Yang MC, Chung TW, Chen JY, Chou NK and Wang SS: Cardiac repair achieved by bone marrow mesenchymal stem cells/silk fibroin/hyaluronic acid patches in a rat of myocardial infarction model. Biomaterials 33: 5541-5551, 2012.

8. Huang B, Qian J, Ma J, et al: Myocardial transfection of hypoxia-inducible factor-1alpha and co-transplantation of mesenchymal stem cells enhance cardiac repair in rats with experimental myocardial infarction. Stem Cell Res Ther: 5, 22, 2014.

9. Takahashi M, Li TS, Suzuki R, et al: Cytokines produced by bone marrow cells can contribute to functional improvement of the infarcted heart by protecting cardiomyocytes from ischemic injury. Am J Physiol Heart Circ Physiol 291: H886-H893, 2006.

10. Wang Y, Liu XC, Zhang GW, et al: A new transmyocardial degradable stent combined with growth factor, heparin, and stem cells in acute myocardial infarction. Cardiovasc Res 84: 461-469, 2009.

11. Barbash IM, Chouraqui P, Baron J, et al: Systemic delivery of bone marrow-derived mesenchymal stem cells to the infarcted myocardium: feasibility, cell migration, and body distribution. Circulation 108: 863-868, 2003.

12. Grajek S, Popiel M, Gil L, et al: Influence of bone marrow stem cells on left ventricle perfusion and ejection fraction in patients with acute myocardial infarction of anterior wall: randomized clinical trial: Impact of bone marrow stem cell intracoronary infusion on improvement of microcirculation. Eur Heart J 31: 691-702, 2010.

13. Hoehn M, Küstermann E, Blunk J, et al: Monitoring of implanted stem cell migration in vivo: a highly resolved in vivo magnetic resonance imaging investigation of experimental stroke in rat. Proc Natl Acad Sci USA 99: 16267-16272, 2002.

14. Bulte JW and Kraitchman DL: Iron oxide MR contrast agents for molecular and cellular imaging. NMR Biomed 17: 484-499, 2004.

15. Templin C, Zweigerdt R, Schwanke K, et al: Transplantation and tracking of human-induced pluripotent stem cells in a pig model of myocardial infarction: assessment of cell survival, engraftment, and distribution by hybrid single photon emission computed tomography/computed tomography of sodium iodide symporter transgene expression. Circulation 126: 430-439, 2012.

16. Dwyer RM, Schatz SM, Bergert ER, et al: A preclinical large animal model of adenovirus-mediated expression of the sodium-iodide symporter for radioiodide imaging and therapy of locally recurrent prostate cancer. Mol Ther 12: 835-841, 2005. 
17. Pan Y, Liu S, Wu H, Lv J, Xu X and Zhang Y: Baculovirus as an ideal radionuclide reporter gene vector: a new strategy for monitoring the fate of human stem cells in vivo. PLoS One 8: e61305, 2013.

18. Grünwald GK, Vetter A, Klutz K, et al: Systemic image-guided liver cancer radiovirotherapy using dendrimer-coated adenovirus encoding the sodium iodide symporter as theranostic gene. J Nucl Med 54: 1450-1457, 2013.

19. Li W, Ma N, Ong LL, et al: Bcl-2 engineered MSCs inhibited apoptosis and improved heart function. Stem Cells 25: 2118-2127, 2007.

20. Guo R, Zhang R, Pan Y, et al: Feasibility of a novel positive feedback effect of 131I-promoted Bac-Egr1-hNIS expression in malignant glioma through baculovirus: a comparative study with Bac-CMV-hNIS. Nucl Med Commun 32: 402-409, 2011.

21. Meirelles Lda S and Nardi NB: Murine marrow-derived mesenchymal stem cell: isolation, in vitro expansion, and characterization. Br J Haematol 123: 702-711, 2003.

22. Rombouts WJ and Ploemacher RE: Primary murine MSC show highly efficient homing to the bone marrow but lose homing ability following culture. Leukemia 17: 160-170, 2003.

23. Livak KJ and Schmittgen TD: Analysis of relative gene expression data using real-time quantitative PCR and the 2(-Delta Delta C(T)) Method. Methods 25: 402-408, 2001

24. Weiss SJ, Philp NJ and Grollman EF: Iodide transport in a continuous line of cultured cells from rat thyroid. Endocrinology 114: 1090-1098, 1984.

25. Libby P and Ridker PM: Novel inflammatory markers of coronary risk: theory versus practice. Circulation 100: 1148-1150, 1999.

26. Zhang SN, Sun AJ, Ge JB, et al: Intracoronary autologous bone marrow stem cells transfer for patients with acute myocardial infarction: a meta-analysis of randomised controlled trials. Int J Cardiol 136: 178-185, 2009.
27. Reinecke $\mathrm{H}$ and Murry $\mathrm{CE}$ : Cell grafting for cardiac repair. Methods Mol Biol 219: 97-112, 2003.

28. Cheng Z, Ou L, Zhou X, et al: Targeted migration of mesenchymal stem cells modified with CXCR4 gene to infarcted myocardium improves cardiac performance. Mol Ther 16: 571-579, 2008.

29. Liu XH, Bai CG, Xu ZY, et al: Therapeutic potential of angiogenin modified mesenchymal stem cells: angiogenin improves mesenchymal stem cells survival under hypoxia and enhances vasculogenesis in myocardial infarction. Microvasc Res 76: 23-30, 2008.

30. Kim SH, Moon HH, Kim HA, Hwang KC, Lee M and Choi D: Hypoxia-inducible vascular endothelial growth factor-engineered mesenchymal stem cells prevent myocardial ischemic injury. Mol Ther 19: 741-750, 2011

31. Zeng B, Lin G, Ren X, Zhang Y and Chen H: Over-expression of HO-1 on mesenchymal stem cells promotes angiogenesis and improves myocardial function in infarcted myocardium. J Biomed Sci 17: 80, 2010.

32. Guo R, Ma Y, Zhang R, et al: Rhenium-188 labeled recombinant human plasminogen kringle 5 (rhk5) and preliminary biodistribution. Evaluation in mice bearing A549 tumours. Nuklearmedizin 50: 234-239, 2011.

33. Acton PD and Zhou R: Imaging reporter genes for cell tracking with PET and SPECT. Q J Nucl Med Mol Imaging 49: 349-360, 2005.

34. Selkirk SM: Gene therapy in clinical medicine. Postgrad Med J 80: 560-570, 2004.

35. Yee JK and Zaia JA: Prospects for gene therapy using HIV-based vectors. Somat Cell Mol Genet 26: 159-174, 2001.

36. Nicola JP, Basquin C, Portulano C, Reyna-Neyra A, Paroder M and Carrasco N: The Nat/I- symporter mediates active iodide uptake in the intestine. Am J Physiol Cell Physiol 296: C654-C662, 2009. 